\title{
Researches on the separation of impurities from a seed flow based on the aerodynamic principle
}

\author{
Gheorghe Stroescu, ${ }^{1,}$, Anişoara Păun ${ }^{1}$, Gheorghe Voicu ${ }^{2}$, Carmen Brăcăcescu ${ }^{1}$, Radu Popa ${ }^{1}$ \\ and Vlad Popa $^{1}$ \\ ${ }^{1}$ National Institute of Research - Development for Machines and Installations designed to \\ Agriculture and Food Industry - INMA, Bucharest 013813, Romania \\ ${ }^{2}$ University Politehnica of Bucharest/Faculty of Biotechnical Systems Engineering, \\ Romania
}

\begin{abstract}
Ensuring for production the necessary quantities of biological material of valuable varieties and hybrids to maintain their initial characteristics requires the permanent application of measures to be carried out on a scientific basis, depending on the biological particularities of the variety or hybrid. The production of seeds for leguminous plants is of great importance because the vast majority of species propagate that way. Due to the fact the yield obtained depends on the quality of the seeds, it is necessary to use seeds of varieties and hybrids with superior qualities and from higher biological categories without foreign bodies (impurities). Knowing the particularities of leguminous plant seed is of special practical importance because the conditioning technologies and machinery are based on it and also the culture technologies that are applied both in the field and in protected areas. The paper presents some theoretical aspects regarding the separation of seed mixtures based on the aerodynamic principle (resistance of the seed mass layer to the air passage, seed floating capacity, air flow working rates, total air flow, physical characteristics of seeds, etc.) which were the basis for designing a conditioning module for vegetable, flower and cereal seeds.
\end{abstract}

\section{Introduction}

Producing seeds for leguminous plants is of a major importance, as the majority of species propagate that way. Due to the fact the yield obtained depends on the quality of the seeds, it is necessary to use seeds of varieties and hybrids with superior qualities and from higher biological categories.

Knowing the particularities of leguminous plant seed is of special practical importance because it is the basis of the conditioning technologies and also of the culture technologies (that are applied both in the field and in protected areas). Seed quality is a rather relative concept, due to the fact that some species seeds can have different destinations: food or fodder consumption, sowing or industrialization $[1,2]$.

* Corresponding author: gigistroescu62@gmail.com 
Knowing the aerodynamic characteristics of grains (terminal velocity, drag coefficient) is significant for the construction and operation of machines, which treat substances with air flow, and in all cases when substances are moved in the air $[3,4,5]$. Some studies showed that aerodynamic properties of an agricultural product (in the form of seeds) also changes according to its mass, volume, equivalent diameter, shape and projected area of the product that many researchers have studied for different products at different moisture content $[6,7]$.

There are various researches on the aerodynamic properties of agricultural product, in the form of non-spherical and spherical particles, which considered factor of its form and volume and stated the drag coefficient as a function of Reynolds number [8].

The paper presents some theoretical relevant aspects related to the separation of seed mixtures based on difference in aerodynamic properties and the movement of the particles in an air flow, aspects which underlay the elaboration of an modern technology for obtaining the seed material and the design of a Conditioning Module for vegetable, flower and cereal seeds, efficient world-wide, intended for sorting and cleaning seed mixtures [9].

\section{Materials and methods}

During separation, a mass particle $m$ is introduced in the area affected by the air flow (Figure 1) and it moves in this air flow under the action of gravity force $G=m g$ and resistance force $R$.

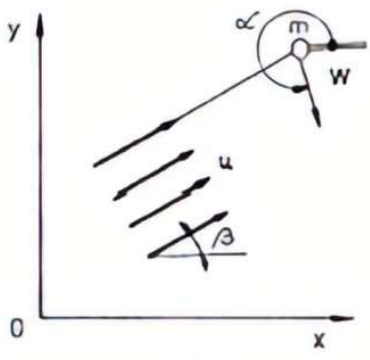

c)

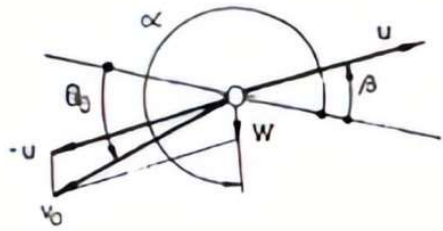

D)

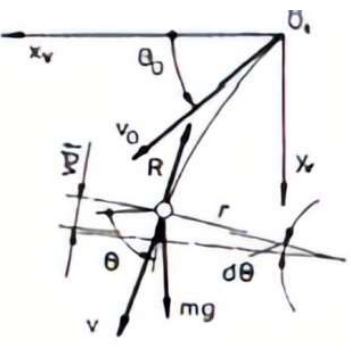

c)

Fig. 1. Scheme of particles movement in a uniform air stream:

a) the introduction of the mass particle $m$ in the interaction zone with the air flow; b) estimating the relative velocity of particle movement $v$ by introducing it in the area affected by the flow; c) the effect of the flow and particle interaction on the relative movement trajectory

The resistance force direction is opposite to the moment of relative velocity $v$ and its value is proportional to the second power of velocity $k v^{2}$. When the particle enters the air flow, it moves to the relative velocity $v$, the value and direction of which are described as the resultant of the parallelogram constructed using the vectors $-\bar{u}$ si $\bar{\omega}$.

From Figure 1 it results that:

$$
v_{0}=\sqrt{u+\omega^{2}-2 u \omega \cos (\alpha-\beta)}, \mathrm{m} / \mathrm{s}
$$

Considering the law of sines, the following can be obtained:

$$
\theta=\beta-\arcsin \left[\frac{\omega}{v_{0}} \sin (\alpha-\beta)\right]
$$

Assuming that the axes of the Cartesian coordinate system move with the air flow, the equations of the particles relative movement become:

$$
\frac{d v}{d t}=g \sin \theta-k v^{2}
$$




$$
\frac{v^{2}}{r}=g \cos \theta
$$

where: $\mathrm{r}$ - radius of curvature of trajectory, $\theta$ - angle between axis $\mathrm{x}^{\mathrm{v}}$ and the tangent to the relative velocity $v$.

Dividing equation (3) by (4) and considering that $v d t=r d \theta$, we get the velocity equations:

$$
\begin{gathered}
\frac{d v}{v^{3} d v}=\frac{\operatorname{tg} \theta}{v^{2}}-\frac{k}{g \cos \theta} \\
-\frac{2 d v}{v^{3} \cos ^{2} \theta}=\frac{k}{g}\left(\frac{2 d \theta}{\cos ^{3} \theta}\right)
\end{gathered}
$$

where: $\mathrm{k}$ - coefficient of velocity.

For an air flow velocity equal to the particles critical velocity we can accept $k=$ constant.

Integrating equation (5) we get:

$$
\frac{1}{v^{2} \cos ^{2} \theta}+c_{2}=\frac{k}{g}\left[\frac{\sin \theta}{\cos ^{2} \theta}+\ln t g\left(\frac{\theta}{2}+\frac{\pi}{4}\right)\right]
$$

The significance of constant $c_{2}$ can be found based on some initial conditions that are: $t=0, v=v_{o}, \theta=\theta_{o}$ as follows:

$$
c^{2}=\frac{k}{g}\left[\frac{\sin \theta_{0}}{\cos ^{2} \theta_{0}}+\operatorname{lntg}\left(\frac{\theta_{0}}{2}+\frac{\pi}{4}\right)\right]-\frac{1}{v_{0}^{2} \cos ^{2} \theta_{0}}
$$

From equations (6) and (7) we obtain a formula that helps to get the relative velocity $v$ according to the angle $\theta$.

$$
v=\frac{1}{\cos \theta \sqrt{\frac{k}{g}\left[\frac{\sin \theta}{\cos ^{2} \theta}+\ln \left(\frac{\theta}{2}+\frac{\pi}{4}\right)\right]-c_{2}}}, \mathrm{~m} / \mathrm{s}
$$

Since the relative velocity $v$ is a quantity of function $\theta$, it results:

$$
t=\int_{\theta_{0}}^{\theta} \frac{d \theta}{g \cos ^{2} \theta \sqrt{\frac{k}{g}\left[\frac{\sin \theta}{\cos ^{2}}+\ln t\right.}\left(\frac{\theta}{2}+\frac{\pi}{4}\right]-c_{2}}
$$

The relative movement is described by the expression:

where

$$
S_{v}=\int v d t=\frac{1}{2 k} \ln \left\langle\left[\frac{\sin \theta}{\cos _{\theta}^{2}}+\ln t g\left(\frac{\theta}{2}+\frac{\pi}{4}\right)\right]-c_{2}\right\rangle-c_{3}
$$

$$
c_{3}=\frac{1}{2 k} \ln \frac{1}{v_{0}^{2} \cos ^{2} \theta_{0}}
$$

At this stage we can estimate the particle transition during the relative movement with reference to the Cartesian coordinate system as follows:

$$
\begin{gathered}
x_{v}=\int v \cos \theta d t=\int_{\theta_{0}}^{\theta} \frac{d \theta}{g \cos ^{2} \theta\left(\frac{k}{g}\left[\frac{\sin \theta}{\cos _{\theta}^{2}}+\ln \left(\frac{\theta}{2}+\frac{\pi}{4}\right)\right]-c_{2}\right\rangle} \\
y_{v}=\int v \sin \theta d t=\int_{\theta_{0}}^{\theta} \frac{\operatorname{tg} \theta d \theta}{g \cos ^{2} \theta\left(\frac{k}{g}\left[\frac{\sin \theta}{\cos _{\theta}^{2}}+\ln t g\left(\frac{\theta}{2}+\frac{\pi}{4}\right)\right]-c_{2}\right\rangle}
\end{gathered}
$$

Thus, the equations of relative movement of the particles under unrestricted air flow and resistance forces can be described as follows:

$$
\begin{aligned}
& \frac{d v}{d t}=-k v^{2} \\
& \frac{v^{2}}{r}=0
\end{aligned}
$$

From equation (14), after integration, we get the expression for $v$ : 


$$
v=\frac{v_{0}}{1+v_{0} k t}, \mathrm{~m} / \mathrm{s}
$$

But the value of $v_{0}$ has to be calculated from equation (1).

From equation (15) it results that $r=\infty$ and it means that $\theta=$ cons and that the trajectory of the particle relative movement is a straight line. If the coefficient of volatility $k$ is constant., we can easily obtain the expression that estimates the projection of the particle movement in absolute movement:

$$
\begin{gathered}
\frac{d x}{d t}=u \cos \beta-\frac{v_{0} \cos \theta}{1+v_{0} k t} \\
\frac{d y}{d t}=u \sin \beta-\frac{v_{0} \sin \theta}{1+v_{0} k t}
\end{gathered}
$$

where:

$$
\theta=\beta-\arcsin \left[\frac{\omega}{v_{0}} \sin (\alpha-\beta]\right.
$$

By integrating equations (17) and (18) we obtain:

$$
\begin{aligned}
& x=u t \cos \beta-\frac{\cos \theta}{k} \ln \left|1+v_{0} k t\right| \\
& y=u t \sin \beta-\frac{\sin \theta}{k} \ln \left|1+v_{0} k t\right|
\end{aligned}
$$

\section{Results and discussions}

Equations (19) and (20) can be used in pneumatic separation processes, especially when calculating the movement parameters of the various components in the initial seeds mass, although air flow velocity may be several times higher than the critical velocity of the particles to be separated and sorted. It should be emphasized that those mathematical expressions have non-linear interdependencies and is virtually impossible to finding an analytical solution. Thus, in order to solve them practically, the transformation into computerized modelling is necessary.

It can be concluded that in order to carry out the separation process by aerodynamic proprieties of a mixture of solid particles (seeds in particular) one has to take into account a series of factors that have a major impact on the way of its development, through which it is highlighted $[1,10,12,15]$

- properties of solid particles subjected to aerodynamic separation process;

- air flow direction and flow regime;

- mode of realization of the biphasic gas-solid mixture (G-S).

The resistance of the product mass layer to the passage of air shall be calculated with the relation:

$$
R=A \cdot h \cdot W^{n}, \mathrm{mmH}_{2} \mathrm{O}
$$

where: $\quad h$ - thickness of product layer, in m;

$W$ - conventional air velocity relative to the whole section of the seed layer, in $\mathrm{m} / \mathrm{s}$;

$A, n$ - coefficients determined experimentally according to the physical characteristics of the seeds.

The aerodynamic properties of the seeds have not yet been determined with sufficient precision since they depend on a number of factors, the mutual connection of which cannot be taken into account with sufficient precision.

The floating velocity depends on the specific weight, the surface condition, the shape and geometric dimensions of the particles of the seed mixture.

Table 1 shows, according to the literature $[2,3,6]$, the aerodynamic properties for several types of agricultural products (in the form of seeds or grains). 
Table 1. Aerodynamic characteristics for several types of agricultural products

\begin{tabular}{|l|c|c|c|}
\hline \multirow{2}{*}{ Product } & \multicolumn{3}{|c|}{ Aerodynamic properties } \\
\cline { 2 - 4 } & $\begin{array}{c}\text { Specific weight } \\
\left(\mathbf{k g} / \mathbf{m}^{\mathbf{3}}\right)\end{array}$ & $\begin{array}{c}\text { Aerodynamic drag } \\
\text { coefficient }\end{array}$ & $\begin{array}{c}\text { Floating velocity } \\
(\mathbf{m} / \mathbf{s})\end{array}$ \\
\hline Wheat & 1.216 & $0.184-0.265$ & $8.9-11.5$ \\
\hline Rye & 1.210 & $0.16-0.22$ & $8.3-9.89$ \\
\hline Barley & 1.200 & $0.191-0.272$ & $8.08-9.11$ \\
\hline Maize & 1.238 & $0.16-0.24$ & $12.48-14.03$ \\
\hline Soybean & 1.092 & $0.115-0.152$ & $17.25-20.16$ \\
\hline Pea & 1.264 & $0.190-0.229$ & $15.50-17.50$ \\
\hline Bean & 1.220 & $0.159-0.259$ & $12.48-14.03$ \\
\hline
\end{tabular}

For precleaning or cleaning, the air flow can be determined with the relation:

$$
Q_{a p}=k_{u} \cdot S_{c p} \cdot v_{a 1}, \mathrm{~m}^{3} / \mathrm{s}
$$

while for sorting, it can be determined with the relation:

$$
Q_{a s}=k_{u} \cdot S_{c s} \cdot v_{a 2}, \mathrm{~m}^{3} / \mathrm{s}
$$

where: $k_{u}$ is the coefficient of the air flow on the sieve $\left(k_{u}=0.65\right)$;

$v_{a 1}$ and $v_{a 2}$ - air stream velocities, $\mathrm{m} / \mathrm{s}$;

$S_{c p}$ and $S_{c s^{-}}$sections of channels for the transport of separated fractions, $\mathrm{m}^{2}$ that are determined by relations:

$$
\begin{array}{cc}
S_{c p}=\frac{Q \cdot\left(100-q_{c p}\right)}{100 \cdot q_{s}} & , \mathrm{~m}^{2} \\
S_{c s}=\frac{Q \cdot\left(100-q_{c p}-q_{c s}\right)}{100 \cdot q_{s}} & , \mathrm{~m}^{2}
\end{array}
$$

where: $Q$ is the feed flow rate of the installation, $\mathrm{m}^{3} / \mathrm{s}$;

$q_{c p}$ and $q_{c s}$ flow rates of the fractions that must be eliminated;

$q_{s^{-}}$product (seed mixture) specific feed rate for an installation that is supplied with an air flow rate of $1 \mathrm{~m}^{3} / \mathrm{s}$.

The total air flow rate $\left(\mathrm{m}^{3} / \mathrm{s}\right)$ required by suction cleaning separator can be determined by the relation:

$$
Q_{a}=\sum_{i=1}^{n} Q_{s i}+\sum_{i=1}^{n} Q_{t i}, \mathrm{~m}^{3} / \mathrm{s}
$$

where:

$\mathrm{Q}_{\text {si }}$ is the air flow rate required for impurities separation in step $\mathrm{i}$ (for an installation with n separation steps);

$\mathrm{Q}_{\mathrm{ti}}$ - the air flow rate required to transport the product (seed mass) from step $i$ to the next step.

Seed conditioning module for leguminous species MCSL, designed and made by INMA Bucharest can perform both the cleaning of vegetable seeds from light impurities and the sorting of the product, according to the technological flow diagram in Figure 2. 


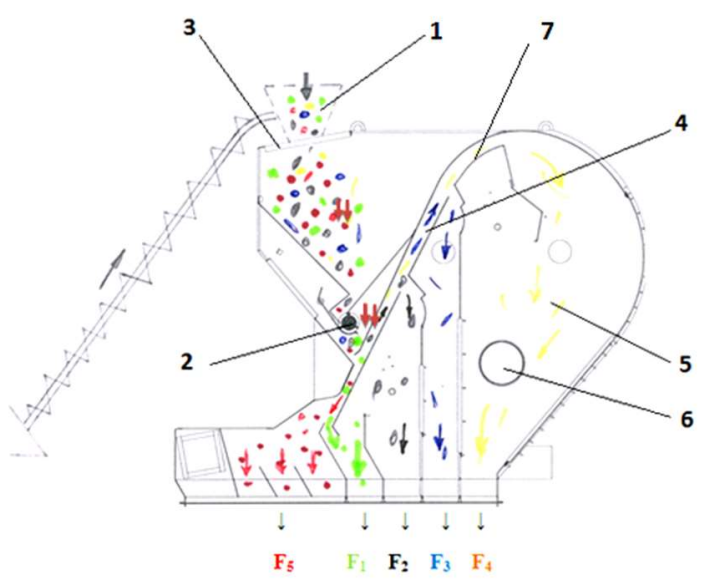

Fig. 2. Technological flow scheme of seed conditioning module MCSL

The product (seed mass) is brought into the feed funnel (1) from where it is distributed evenly through the sorting column (4) by means of cylinder (2) and the feed flap (3). Under the influence of the ascending air flow produced by the fan (6) within the floating velocity limits, the heavier bodies fall to the bottom obtaining fraction $F_{1}$, while the lighter ones rise to the first orifice where fraction $F_{2}$ is separated, to the second orifice where the fraction $F_{3}$ is separated or leaves the column at the top entering the decanting chamber (5) where $\mathrm{F}_{4}$ fraction of light bodies (impurities) is deposited. The sucked air from the decanting chamber is fed by the fan into the second decanting chamber where dust and other very light impurities are separated forming the fraction $F_{5}$. The adjustable wall (7) is designed to modify the sorting column section (4) to influence the ascending air flow velocity for seed sorting through four separation windows.

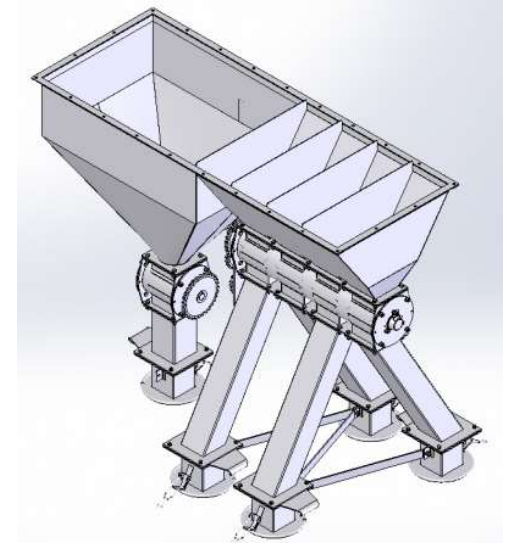

Fig. 3. 3-D representation of the sorts discharge system

\section{Conclusions}

In order to achieve the separation of a solid particles (heterogeneous mixture) in good conditions it is necessary to know, for each component of the mixture, their properties;

The sorting of solid particle mixtures according to the aerodynamic properties of the components is used in order to increase the purity degree of the basic product;

The aerodynamic separation process is a complex process due to the fact that it is influenced by a number of factors: 
- the physico-mechanical characteristics of the solid particles contained in the heterogeneous mixture subjected to the aerodynamic separation process;

- air flow direction;

- constructive and functional characteristics of the installation performing the aerodynamic separation.

The MCSL module made by INMA Bucharest is designed to improve the seed conditioning process, an operation performed only by using air flow.

\section{Acknowledgements}

This work was financed with:

- the support of the Romanian Ministry of Research and Innovation, Programme P1 Development of the National R \& D system; Subprogram 1.2. Institutional Performance Institutional Development Projects - Complex Projects Realized in RDI Consortia, project: FUTURE FARMING ENSURING ENVIRONMENTAL SUSTAINABILITY BY INCREASING IMPROVEMENT COMPETITIVENESS FOR ORGANIC FARMING, Contract: 14PCCDI/06.03.2018), project component 4 "Making efficient the technology of producing ecological seeds for legume species by achieving a module of seed conditioning"- INMA Bucharest,

- a grant of the Romanian Ministry of Research and Innovation, through Programme 1 Development of the national research-development system, subprogramme 1.2 - Institutional performance - Projects for financing excellence in RDI, contract no. 16PFE.

\section{References}

1. N. Bucurescu, D. Roman, Seed and its preparation for sowing, Ceres Publishing House, Bucharest (1992)

2. L. Ioancea - Conditioning and superior capitalisation of plant raw materials for food purposes. Technology and installation, Ceres Publishing House, Bucharest (1988)

3. B Y. Gorial, J R. O' Callaghan, Journal of Agricultural Engineering Research, 46(3), (1990).

4. R.K. Gupta, G. Arora, R. Sharma, , Journal of Food Engineering, 79, (2007)

5. A.D. Zewdu, Biosystems Engineering, 98, (2007)

6. P. H. Mortensen, H. I.Andersson, J. J. J. Gillissen and B. J Boersma., Physics of fluids, 19, (2007)

7. E. Moşneguțu, Contributions to the aerodynamic sorting of agricultural products, "Gh. Asachi" Technical University of Iaşi (2006)

8. NN. Mohsenin, Physical Properties of Plant and Animal Materials (Handbook). (2nd ed.). Gordon \& Breach (1986)

9. A. Păun, C. Brăcăcescu, D. Milea, G. Bunduchi, Proceedings of 16-th International Scientific Conference Engineering for Rural Development, Jelgava, Latvia (2017) 